\title{
RESCATE DEL CONOCIMIENTO ANCESTRAL DE UN PATRIMONIO AGROALIMENTARIO Y FORESTAL DE LA REGIÓN DE ATACAMA VALORACIÓN NUTRICIONAL DEL FRUTO Y ARROPE DE CHAÑAR Geoffroea decorticans (Gill. ex Hook. et Arn.)
}

\author{
Gacitúa, S $^{12}$; González, M. ${ }^{12}$; Muñoz, R. ${ }^{13}$; Villalobos, E. ${ }^{12}$ y Montenegro, J. ${ }^{12}$
}

\section{RESUMEN}

El chañar (Geoffroea decorticans Gill. ex Hook. et Arn.) es una especie nativa arbórea de la familia Fabaceae que crece en la Región de Atacama, Chile, y en países limítrofes. Esta especie produce frutos que han sido fuente de alimento ancestral y étnico, constituyendo un importante patrimonio alimentario y cultural. El fruto del chañar tiene un alto potencial nutracéutico aportado por el contenido de polifenoles, como son los flavonoides, y también por la actividad antioxidante, permitiendo de esta manera promover el consumo del fruto y derivados, sea como arrope $u$ otros productos de chañar.

En este contexto, el objetivo de estudio fue rescatar el conocimiento tradicional ancestral y valorar el patrimonio alimentario del fruto y arrope de chañar, con el fin de evidenciar su uso alimenticio a través del arrope de chañar como un producto con características especiales para consumidores que buscan lo natural y lo orgánico, y su connotación étnica y ancestral.

Para el rescate del conocimiento ancestral referido al uso y proceso productivo se usaron procedimientos metodológicos que se enmarcan en estrategias y técnicas cualitativas, las que fueron aplicadas y trabajadas con las comunidades Collas y la Red de Mujeres Rurales e Indígenas (RATMURI), ambos de la Región de Atacama. Para la valoración nutricional se determinaron los componentes proximales, antioxidantes y flavonoides de frutos de chañar, específicamente del mesocarpio (pulpa s/cáscara) y su semilla (s/carozo), colectados de diferentes sectores de la Comuna de Copiapó.

Los resultados indican alta presencia de carbohidratos, de flavonoides y de antioxidantes, lo que unido a lo que la literatura indica, posiciona los frutos y el arrope del chañar como un importante producto, no solamente dirigido hacia el ámbito del consumo de alimentos en mercados nichos sino también en el ámbito de la medicina popular (producto etnofarmacéutico).

Palabras claves: Chañar, Geoffroea decorticans, Componentes alimenticios, Producto étnico, Producto ancestral, Productos forestales no madereros.

\footnotetext{
12 Investigadores, Instituto Forestal sgacitua@infor.cl

${ }^{13}$ Investigador, Facultad de Agronomía, Universidad de Concepción.
} 


\section{SUMMARY}

Chañar (Geoffroea decorticans Gill.ex Hook. et Arn.) is a native tree of the Fabaceae family growing at the Atacama Region in Chile and also in bordering countries. Chañar fruits are an ethnic and ancestral food source and represent an important nutritional and cultural heritage. The fruits have a high nutraceutical potential because of their polyphenols contents, such as flavonoids, and also due to its antioxidant properties, allowing that way the promotion of the fruits and derivatives consumption as arrope or other products.

The main objective of the study is to recover the traditional and ancestral knowledge and to value the Chañar fruit nutritional patrimony, in order to demonstrate it use as especial properties product to consumers who like natural and organic alternatives as well as their ethnic and ancestral connotation.

To rescue the ancestral knowledge on the use and productive process methodological procedures based on technical and qualitative strategies were used to work with the Collas communities and the Women Rural and Indigenous Net (RATMURI) in the Atacama Region. To the fruit nutritional valuation proximal components, antioxidants and flavonoids were determined by using mesocarp (pulp without skin) and seeds collected from areas of the Copiapó Commune.

The results show high carbohydrates, flavonoids and antioxidants presence and these characteristics, together wit the bibliographic information, position fruits and arrope as important products, not only in the food consumption field in niche markets but also in the popular medicine field (ethno-pharmaceutical product)

Key words: Chañar, Geoffroea decorticans, Nutritional components, Ethnic products, Ancestral products, Non wood forests products. 


\section{INTRODUCCIÓN}

Los Productos forestales no madereros (PFNM) son bienes de origen biológico distintos de la madera, derivados del bosque, de otras áreas forestales y de los árboles fuera de los bosques. Los PFNM pueden recolectarse en forma silvestre o producirse en plantaciones forestales o sistemas agroforestales (FAO, 1999).

Los frutos de chañar son productos forestales no madereros y su importancia radica en que pueden constituirse en un importante ingreso económico para las comunidades rurales, en particular para aquellas que habitan las zonas en donde crece esta especie, donde representa una fuente de alimentos para los pueblos originarios, enraizada a su cultura, y una potencial fuente de desarrollo de la comunidad de productores que la cultivan y hacen uso del producto y sus derivados.

El chañar pertenece a la familia Fabaceae, es una especie arbórea silvestre que se encuentra difundida en la zona norte del país y, por su adaptación a zonas áridas, tiene un alto potencial de uso en el combate de la desertificación y además sus frutos tienen propiedades alimenticias y medicinales, son utilizados como forraje para el ganado caprino y las comunidades locales también los emplean en la elaboración de un jarabe conocido como arrope.

Los frutos del Chañar son drupáceos, ovoides o globosos, pardo-rojizo a la madurez y constituyen una oportunidad para ampliar estudios dirigidos a revalorizarlo como un recurso alimenticio ancestral y étnico, si se los considera como fuente de ingredientes para elaborar productos con potencial direccionamiento a mercado gourmet, e incluso profundizar la búsqueda en sus constituyentes, que lo harían alcanzar la connotación de fuente de alimentos nutraceuticos o funcionales.

Sobre la base que el chañar produce frutos que han sido fuente de alimento ancestral y étnico de la Región de Atacama, y que constituye un patrimonio cultural posible de recuperar, este trabajo tuvo como objetivo rescatar el conocimiento tradicional ancestral y valorar el patrimonio alimentario del fruto y arrope de chañar, colectados y elaborados por las propias comunidades rurales e Indígenas, que hacen uso de ellos, con el objeto de hacer conocido un producto con uso alimenticio con características especiales para consumidores que buscan lo natural, orgánico, étnico y ancestral.

\section{MATERIAL Y MÉTODO}

\section{Rescate del Conocimiento Ancestral}

Para el rescate del conocimiento ancestral referido al uso y proceso productivo se utilizaron técnicas metodológicas cualitativas, las que fueron aplicadas y trabajadas con la comunidad Colla Piedra Luna, del sector Piedra Colgada, y la Red de Mujeres Rurales e Indígenas (RATMURI) del sector de San Pedro, ambos de la Región de Atacama. Para ello, se elaboró de manera conjunta la matriz de localización, se diseñó y aplicó una serie de entrevistas semiestructuradas diferenciadas a recolectores, propietarios, productores e informantes institucionales, la que permitió recoger información en tres aspectos: Origen y evolución histórica del producto, traspaso de información respecto al proceso de elaboración dentro de las familias (método genealógico) y proceso ancestral de elaboración de arrope.

Adicionalmente, junto a los elaboradores de chañar en cada comunidad, se realizó un estudio de campo (observación participativa) de la elaboración del arrope, lo que permitió favorecer el rescate de la información referida al uso y proceso productivo además de las relaciones entre el sistema social y el ecológico, resultando esto fundamental para establecer niveles de identidad territorial actual de la comunidad junto con identificar acciones o procesos determinantes en su construcción, además de reconocer variables que pudieran ayudar en su reconocimiento, tanto en el ámbito de diferenciación (lo que los distingue) así como en su proyección (la voluntad de quiénes quieren llegar a ser). 


\section{Origen y Colecta de Material Frutal}

Se trabajó en rodales naturales de chañar distribuidos en tres sectores de la cuenca del rio Copiapó, Comuna de Copiapó, usando la cercanía al mar como referencia en la comuna del mismo nombre en Piedra Colgada (lejano); Barranquilla (intermedio) y Costa (contiguo). De cada sector se seleccionaron 10 árboles que presentaban características saludables y una altura entre 1,5 y $3 \mathrm{~m}$, con el propósito de trabajar con árboles relativamente jóvenes y de edades similares. Durante el mes de febrero del 2017 se procedió a colectar frutos maduros de cada árbol por sector, de forma manual y en conjunto con las comunidades.

\section{Elaboración de Arrope de Fruto de Chañar}

A partir de los frutos colectados en cada sector, tanto las comunidades Collas como la Red de Mujeres Rurales e Indígenas (RATMURI) elaboraron sus propios arropes según métodos ancestrales-artesanales, obteniéndose un total de seis muestras de arrope. Con posterioridad se realizó una reunión de discusión, donde se logró consensuar el proceso de elaboración utilizado actualmente por ambas comunidades.

\section{Material Analizado}

Muestras de material frutal y de arrope fueron sometidas a análisis químico-nutricional, se remitieron las muestras al laboratorio "Labalimentos" del Departamento de Ingeniería de Alimentos de la Universidad de La Serena. Para el caso de los frutos, las muestras fueron específicamente del mesocarpio (pulpa s/cáscara) y su semilla (s/carozo), colectadas de tres sectores de la Comuna de Copiapó. En el caso del arrope fueron seis muestras elaboradas a partir del fruto de estas tres localidades por ambos grupos.

Las variables analizadas fueron los componentes proximales, capacidad antioxidante y contenido de flavonoides. Las metodologías analíticas aplicadas corresponden a las oficiales de la AOAC (Association of Official Agricultural Chemists, USA) para los componentes mayoritarios; proteínas mediante el método Kjeldalh gravimétrico (Of. methods of analysis A.O.A.C. 960.52); grasas totales por el Método Soxhlet Gravimétrico (Of. Methods of Analysis A.O.A.C. 920.39); hidratos de carbono por diferencia, humedad (método AOAC 920.15 gravimétrico); fibra cruda (método AOAC 962.09); digestión básica y acida, azúcares totales y reductores mediante el método Acido 3,5 Dinitrosalicilato (3,5 DNS), energía y cenizas por calculo. La capacidad antioxidante se realizó mediante el método ORAC (descrito por Guorong et al., 2009) y flavonoides totales mediante método espectrofotométrico (descrito por Kim et al., 2003).

\section{RESULTADOS Y DISCUSION}

\section{Rescate del Conocimiento Ancestral Referido al Uso y Proceso Productivo}

Comunidad Piedra Luna, Sector Piedra Colgada, Comuna de Copiapó: Como pueblo originario Colla y en virtud de la legislación indígena vigente, a partir de 2002 se gestiona la asignación de tierras para 32 personas y su grupo familiar; ocupando un territorio natural y tradicional de chañares en que se asienta la comunidad. A cada representante de familia le correspondió en comodato (situación aún en trámite de regularización) un sitio de 3 ha, en el cual se encuentran chañares en unidades variables de 500 a 1.000 y más individuos, de diferentes edades, ubicados de preferencia en terrenos planos de escasa pendiente. De las 3 ha, solo una de ellas en promedio es sometida a manejo productivo temporal, donde los comuneros destacan la falta de agua para riego, como el principal factor limitante para el desarrollo de las actividades vinculadas a este recurso y para la forma de residencia y ocupación permanente de los sitios comunitarios asignados. Simbólicamente han creado un icono de identidad que los representa en la sociedad en que viven.

Comunidad RATMURI, sector San Pedro de Copiapó, sector Serranía Poblete, Comuna de Copiapó: Está conformada por un grupo de recolectores y productores de productos 
del Chañar, procedentes de este lugar y de otros del entorno, tales como Tierra Amarilla; Los Loros, El Tranque, Manfla, Copiapó y otros poblados menores. Genealógicamente proceden de diferentes troncos sanguíneos entre los cuales se encuentran collas, diaguitas y mapuches, pueblos reconocidos y validados jurídicamente (Ley Indígena №19593 de 1993, CONADI). Testimonios de arqueólogos y antropólogos regionales señalan, con respecto al colla del lugar "Esta es la única comunidad indígena Colla que remonta su origen desde la parte baja de la Región, más específicamente, en el poblado de San Pedro, muy cerca de la costa, entre Copiapó y Caldera- en donde existen tierras comunitarias".

Como descendientes de pueblos Indígenas, la mayor parte de ellos se ha involucrado en la gestión y la recuperación de tierras comunitarias ancestrales desde 2010, guiados por algunas líderes de la comunidad con el apoyo de algunos profesionales. Se entregaron a comunidades en condición de permanencia de la tenencia de la tierra 1.000 ha, de las cuales 101 ha corresponden a originarios collas y el resto asignados a otros pueblos reconocidos por la Ley citada. En total son 50 familias que ocupan en forma transitoria estos sitios, ya que la mayor parte vive en Copiapó. Los sitios se encuentran localizados en la parte baja de la Quebrada de Monardéz; ocupando el sector identificado como Serranía Poblete. Esta localización geográfica no ha sido adecuada para el asentamiento de la comunidad, por ser área de riesgo y vulnerabilidad natural; últimamente ha recibido el impacto de dos aluviones (2015 y 2017), provocando pérdidas de infraestructura; de animales y de chañares.

Origen y evolución histórica del producto: Al igual que en el pasado, la comunidad Colla Piedra Luna es recolectora y productora, y a la vez comercializa los productos derivados del chañar, pero de manera informal y por referencias de familiares o amistades. Se señala que estas actividades son familiares, centradas en los sitios que pertenecen a la comunidad. Para RATMURI, se observa una actitud generalizada de respeto hacia la palabra de la mujer mayor y líder de grupo, característica vinculada a una forma social de matriarcado en comunidades aborígenes, muy marcada por los historiadores y antropólogos de la región, que le asignan a los hombres de la comunidad labores en minería y en tiempos más actuales a servicios urbanos.

Traspaso de información respecto al proceso de elaboración dentro de las familias (método genealógico): La comunidad Colla Piedra Luna genealógicamente procede de un tronco sanguíneo colla reconocido y validado jurídicamente (Ley Indígena №19593 de 1993. CONADI). Esta comunidad está representada en la actualidad por tres ramas entrelazadas por vínculos sanguíneos, cuya base familiar es Cordero - Barrera.

De acuerdo a testimonios y experiencias acerca del pasado, la comunidad Piedra Luna identificada como colla se caracteriza por tener una trayectoria de vida en sitios geográficos ocupados por plantas de chañares y se constata la transmisión oral de la utilización de los productos desde generaciones ancestrales. En la actualidad la generación mayor bordea los 80 años (emisores de la tradición) y los involucrados en las actividades vinculadas al recurso promedia los 46 años (receptores de la tradición). Antiguamente, niños y adolescentes se vinculaban a la recolección de frutos y gustaban de participar del procesamiento de los productos, hoy son más lejanos, por razones de estudio y/o trabajo en lugares más distanciados, y por el deseo de sus padres de mejorar su calidad de vida a través del acceso a la educación formal, que en muchos casos significa estudiar en la ciudad, por sobre su permanencia en el campo.

El relato testimonial de la comunidad RATMURI (Serranía Poblete, San Pedro) indica que es más heterogénea que la comunidad Colla Piedra Luna, se caracteriza actualmente por tener una procedencia y trayectoria de vida en sitios geográficos muy diferentes, incluso localizados fuera de la Región de Atacama. El contacto con vecinos y familiares locales integra a los foráneos a una comunidad que siente al chañar como un recurso local y los entusiasman en utilizar el fruto para elaborar arrope (con el tiempo se detectan otras opciones de utilización del mismo). Socialmente, el vínculo matrimonial o de convivencia entre personas de origen colla local con otras etnias constituye un fuerte factor de unión y difusión de los procedimientos que se realizan en la elaboración del arrope y otros subproductos del chañar, que no distan de los realizados en la actualidad. Existen pocos collas que heredan el apellido ancestral, señalando que los apellidos de origen indígena fueron siendo reemplazados por los de la población local que vivía en la zona. 
Proceso tradicional de elaboración de arrope: Tradicionalmente las mujeres han jugado un rol preponderante en el procesamiento de los productos, comportamiento que en estos días se mantiene y es fácil de visualizar en el proceso de elaboración del arrope y otros, no obstante, manifiestan algunas diferencias en detalles de la manipulación de este, como en cantidades de agua y obtención de subproductos como harina poco refinada, mazacote, chicha, aguardiente o simple comida para cerdos. Se observa una actitud generalizada de respeto hacia la mujer mayor y dueña de la tradición, característica vinculada a una forma social de matriarcado típica de las comunidades indígenas. En relación al procesamiento tradicional del arrope, que ocupa varias horas (aproximadamente ocho horas), indican que en el pasado se utilizaban pailas de cobre para la cocción, ya que eso aceleraba el proceso, pero que esto hoy no es factible por el precio elevado de estos artefactos. En tiempo más actuales se usaron incluso tambores de lata. Como el quehacer de elaborar arrope durante la estación es limitada, la familia no realizaba esta elaboración más de una vez por año y la producción era destinada principalmente al consumo familiar. Algunas mujeres también elaboraban harina y, a instancias de los varones, también chicha.

La opinión unánime es que el tiempo ocupado para la elaboración de estos productos no es compensado por el valor que se obtiene de su comercialización, opinión fundamentada en parte por el volumen reducido de producto obtenido del procesamiento versus el tiempo ocupado y el precio en el mercado informal del cual forman parte. Reconocen no tener claridad en cuanto al valor de los productos en el mercado formal y existen además otras comunidades de localizaciones vecinas al sector, no necesariamente de origen colla, que son recolectoras y/o compradoras de frutos y productoras especialmente de arrope y en menor medida harina, con quienes compiten en el mercado informal. Estiman que en las condiciones actuales con carencia de agua (principal amenaza) y poca colaboración de instituciones de apoyo para mejorar su forma de manejo y conservación del recurso, no es factible ampliar el mercado a lo menos del arrope.

Al igual que en Piedra Luna, las mujeres socias de RATMURI han jugado un rol preponderante en la recolección y el procesamiento de los productos, comportamiento que en estos días se visualiza en el proceso de elaboración del arrope y otros; no obstante, manifiestan algunas diferencias en detalles de la manipulación de este, como en cantidades de agua y tiempo ocupado en la cocción, y obtención de los subproductos como harina, galletas y licor.

En relación al procesamiento del arrope, todos señalan que la recolección del chañar por temporada y la producción de arrope y otros productos de chañar, se inicia en los sectores más altos hacia la cordillera (familias oriundas de Manfla, al igual que lo citado en comunidad Piedra Luna). La tradición asimilada señala que han sido recolectores del fruto y por tradición no han comprado ni vendido semillas y frutos, pero si existen algunas familias que regalan los frutos a familiares y conocidos. El producto estaba, y aún está, destinado al consumo familiar y de algunas amistades, en una forma de comercio informal.

En síntesis, el procesamiento al que se someten los frutos de chañar para la elaboración de arrope se inicia con la cosecha de los frutos, los que se acopian en el lugar en que se realiza la elaboración. Se descartan los frutos deteriorados y seguidamente los frutos seleccionados son lavados con agua limpia y son reservados. Se enciende el fogón con leña (muchas veces también de chañar) y los frutos son dispuestos en fondos para su cocción en agua, los que frecuentemente son rellenados con agua para compensar las pérdidas por evaporación. Terminado el proceso de cocción, los frutos se someten a un proceso de molienda manual que consiste en triturar la pulpa del fruto, separando los carozos de este. La pulpa del fruto se somete a un proceso de estrujado manual, para lo cual se utiliza un paño en donde se vierte el líquido con la pulpa, se recibe el líquido proveniente del estrujado (mosto) en un fondo y el bagazo, que es la pulpa y cáscara de la fruta de la cual se ha extraído el jugo, se reserva para la elaboración de harina gruesa. El mosto se somete a un proceso de evaporación que permite concentrarlo durante un tiempo de 8 horas aproximadamente, hasta alcanzar la consistencia del arrope. Luego de enfriar el mosto concentrado, ya denominado arrope, se envasa normalmente en frascos de $250 \mathrm{cc}$ o en botellas de $3 / 4 \mathrm{~L}$ para acopio, almacenaje y posterior venta. En la Figura $N^{\circ} 1$ se muestran los procesos que constituyen el flujograma productivo de arrope, el cual fue obtenido por observación de campo en las comunidades. 


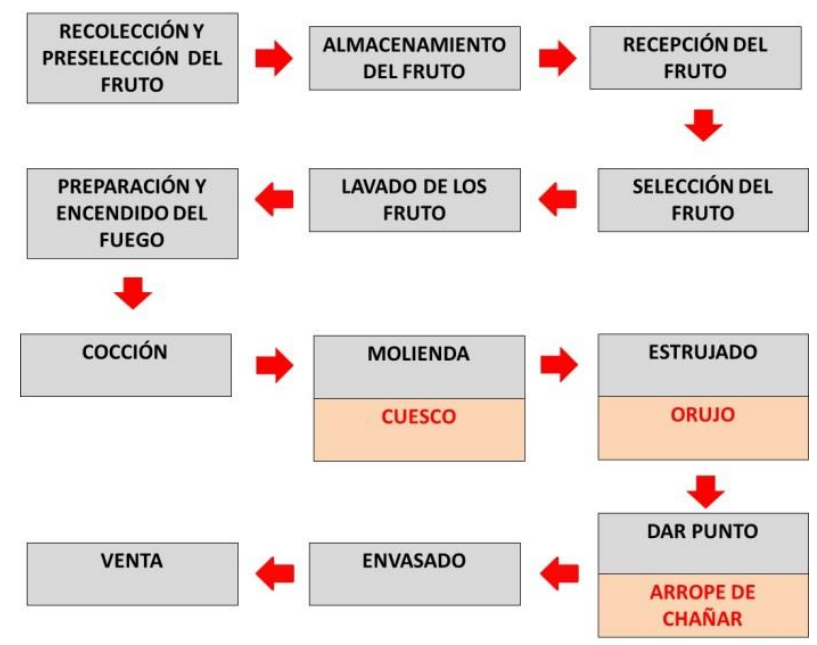

Figura $\mathrm{N}^{\circ} 1$

FLUJO PRODUCTIVO DE LA PRODUCCIÓN DE ARROPE VALIDADO POR LAS COMUNIDADES COLLA PIEDRA LUNA, DEL SECTOR DE PIEDRA COLGADA, Y RATMURI, DEL SECTOR DE SAN PEDRO

\section{Valoración de la Composición Nutricional}

En el Cuadro $\mathrm{N}^{\circ} 1$ se presentan los resultados de las determinaciones de componentes proximales y constituyentes específicos de muestras de pulpa y semillas de frutos provenientes de los tres sectores en estudio, mientras que en el Cuadro $\mathrm{N}^{\circ} 2$ se muestran los resultados de las determinaciones de componentes proximales y constituyentes específicos de las seis muestras de arrope elaborado por las comunidades Piedra Luna y RATMURI, de cada sector en estudio.

Cuadro $\mathrm{N}^{\circ} 1$

COMPONENTES PROXIMALES Y CONSTITUYENTES ESPECÍFICOS DE LAS MUESTRAS DE PULPA Y SEMILLAS PROVENIENTES DE LOS SECTORES DE ESTUDIO

\begin{tabular}{|l|c|c|c|c|c|c|c|c|}
\hline \multirow{2}{*}{ Producto } & \multicolumn{4}{|c|}{ Pulpa } & \multicolumn{4}{c|}{ Semilla } \\
\cline { 2 - 9 } & $\begin{array}{c}\text { Piedra } \\
\text { Colgada }\end{array}$ & Barranquilla & Costa & Promedio & $\begin{array}{c}\text { Piedra } \\
\text { Colgada }\end{array}$ & Barranquilla & $\begin{array}{c}\text { Costa } \\
\text { Previo }\end{array}$ & Promedio \\
\hline Proteínas (\%) & 6,30 & 6,15 & 7,49 & 6,60 & 4,60 & 6,38 & 7,55 & 6,20 \\
\hline Grasa total (\%) & 0,45 & 0,71 & 1,10 & 0,80 & 2,75 & 4,51 & 4,44 & 3,90 \\
\hline $\begin{array}{l}\text { Hidratos de } \\
\text { carbono (\%) }\end{array}$ & 89,0 & 89,0 & 88,0 & 88,7 & 92,0 & 88,0 & 88,0 & 89,3 \\
\hline $\begin{array}{l}\text { Azúcares totales } \\
\text { (\%) }\end{array}$ & 24,0 & 21,0 & 28,0 & 24,3 & 15,0 & 15,0 & 11,0 & 13,7 \\
\hline Humedad (\%) & 15,0 & 15,0 & 15,0 & 15,0 & 12,0 & 10,0 & 10,0 & 10,7 \\
\hline Cenizas (\%) & 6,18 & 4,60 & 4,95 & 5,20 & 1,01 & 1,09 & 0,73 & 0,90 \\
\hline Fibra cruda (\%) & 6,29 & 3,23 & 4,74 & 4,80 & 73,00 & 66,00 & 65,00 & 68,00 \\
\hline Energía (kcal) & 378 & 387 & 392 & 386 & 411 & 418 & 422 & 417 \\
\hline $\begin{array}{l}\text { Capacidad } \\
\text { antioxidante (umol } \\
\text { ET/100g) }\end{array}$ & 1.879 & 2.332 & 2.824 & 2.345 & 2.231 & 2.385 & 3.027 & 2.548 \\
\hline $\begin{array}{l}\text { Flavonoides (mg } \\
\text { CAT/100g) }\end{array}$ & 3.425 & 3.230 & 3.596 & 3.417 & 314 & 176 & 185 & 225 \\
\hline
\end{tabular}


No se observan diferencias importantes en los componentes proximales de pulpa y de semilla provenientes de diferentes sectores, lo que indica que los frutos, sin importar su origen, se podrían utilizar para la elaboración de productos a mayor escala productiva. Aun cuando lo informado por diversos autores no permite que los resultados obtenidos de los componentes proximales de pulpa del fruto, y semillas sean comparados directamente, debido a diferencias de la constitución de la muestra de análisis, De manera orientadora se puede indicar que los contenidos de proteínas, grasas, cenizas e hidratos de carbono de los componentes pulpa, se encuentra en los rangos de los valores informados por Orrabalis et al. (2013) para fruto entero de chañar, que señalaron valores en base seca que oscilan entre 7 y $10 \% ; 1,5$ y $4,9 \% ; 2,5$ y $3,7 \%$, y 81,9 y 86,2 $\%$, respectivamente, según temporada de cosecha.

Se registraron altos valores de carbohidratos y bajos niveles de grasa para todas las muestras de pulpa, los que pueden ser comparados con lo obtenido por Orrabalis et al. (2014) para pulpa de frutos, donde se informa alto contenido de carbohidratos $(85,6 \%)$ y bajo contenido de grasa $(0,9 \%)$ con valores de $9,4 \%$ y $4,2 \%$ para proteínas y cenizas, respectivamente. Los altos contenidos de carbohidratos y de azúcares totales de los componentes de la pulpa del fruto ofrecerían posibilidades de uso para la preparación de productos alimenticios del chañar, del mismo modo los altos valores de azúcares y bajo niveles de grasa, permitirían disponer de una materia prima deseable para elaborar jarabes y mermeladas, entre otros productos.

Se observa que el resultado obtenido del contenido de grasa de la semilla sin endocarpio está muy por debajo de lo que Masson (2012) determinó para muestras de chañar de la Región de Atacama y la Región de Coquimbo, obteniendo contenidos promedios de proteínas y grasas de la semilla de $27,3 \%$ y $47,5 \%$, respectivamente, por otro lado Nogués et al (2013) señalan un contenido de proteína de $21,6 \%$ y de aceite de $48,8 \%$ para semillas sin endocarpio, lo que posiblemente se deba a factores de manejo e historia de las muestras de semillas analizadas, implicando que sería importante realizar mayores estudios de la especie en Chile, en particular en la Región de Atacama, con el fin de explorar cantidad y tipo de ácidos grasos que pueden constituir fuente para fines alimenticios como también para biocombustibles. Aunque no se encuentran datos en literatura sobre contenido calórico de componentes de fruto y arrope de chañar, se puede indicar que varía entre 3,9 y $4,2 \mathrm{kcal} / \mathrm{g}$, según sea pulpa o semilla del fruto, registrando un comportamiento promedio de $4,0 \mathrm{kcal} / \mathrm{g}$ y $2,0 \mathrm{kcal} / \mathrm{g}$ según sean partes del fruto y arrope, respectivamente.

No se ha encontrado referencias que informen sobre los componentes proximales del arrope. Sin embargo, en el Cuadro $\mathrm{N}^{\circ} 2$ se observan valores promedio similares, para los arropes provenientes de las dos comunidades (Piedra Luna y San Pedro), lo que se debería a un grado de homogeneidad de la condición inicial de los frutos, en particular su condición de madurez y que permitiría deducir que esta variable no generaría diferencias en la composición del producto final. Se observa que el arrope proveniente de las comunidades Piedra Luna y San Pedro del Sector Costa, registraron valores de contenido humedad mayores, de 71 y $55 \%$, respectivamente, cuando se compara con los otros dos arropes, en particular el elaborado por la comunidad Piedra Luna, de $41 \%$ y $43 \%$, respectivamente, lo que indicaría posiblemente algunas diferencias en la forma de conducir el proceso de elaboración del arrope, en particular las temperaturas alcanzadas en los tiempos de cocción y la duración de los procesos, lo que debe ser considerado para la vida útil del producto terminado.

Se observa un incremento en la capacidad antioxidante y un detrimento de flavonoides de los arropes (Cuadro $\mathrm{N}^{\circ} 2$ ) cuando se compara con los niveles que presentaron los contenidos en la pulpa (Cuadro $\mathrm{N}^{\circ} 1$ ). Aunque no se encuentran datos en la literatura sobre capacidad antioxidante y de flavonoides en arrope, existe información que indica que las condiciones de cocción (Anwar et al., 2008; Mazzeo et al., 2011), en especial la temperatura, favorecen la ruptura de constituyentes con potencial actividad antioxidante y que el tiempo de cocción por hervido disminuye la capacidad antioxidante y el contenido de polifenoles totales, que correspondería a la situación observada en este caso. Además, Martins et al., (2018) señalan que una alta actividad antoxidante total en jugos concentrados se puede deber a la liberación de fitoquímicos unidos desde la matriz con el procesamiento termal o el efecto aditivo entre fitoquímicos (Dewanto et al., 2002). Esto, puede llevar a plantear estudios acerca de la dinámica de los antioxidantes en el 
arrope que, a diferencia de en otros concentrados de frutas, se deriva de una línea de producción que conlleva un proceso de cocción de frutos y agua agregada.

\section{Cuadro $\mathrm{N}^{\circ} 2$ \\ COMPONENTES PROXIMALES Y CONSTITUYENTES ESPECÍFICOS DE LAS MUESTRAS DE ARROPE ELABORADAS POR LAS COMUNIDADES PIEDRA LUNA (PL) Y RATMURI (RAT) PROVENIENTES DE LOS SECTORES DE ESTUDIO}

\begin{tabular}{|c|c|c|c|c|c|c|c|c|c|}
\hline \multirow{2}{*}{ Producto } & \multicolumn{2}{|c|}{ Piedra Colgada } & \multicolumn{2}{|c|}{ Barranquilla } & \multicolumn{2}{|c|}{$\begin{array}{c}\text { Costa Previo } \\
\text { Santa Margarita }\end{array}$} & \multicolumn{2}{|c|}{$\begin{array}{c}\text { Promedio } \\
\text { Comunidades } \\
\end{array}$} & \multirow{2}{*}{$\begin{array}{l}\text { Promed } \\
\text { io Total }\end{array}$} \\
\hline & PL & RAT & PL & RAT & PL & RAT & PL & RAT & \\
\hline Proteínas (\%) & 1,98 & 1,58 & 2,05 & 1,92 & 1,60 & 1,60 & 1,90 & 1,70 & 1,80 \\
\hline Grasa total (\%) & 0,04 & 0,03 & 0,02 & 0,21 & 0,03 & 0,31 & 0,09 & 0,20 & 0,10 \\
\hline $\begin{array}{l}\text { Hidratos de carbono } \\
(\%)\end{array}$ & 57,0 & 53,0 & 55,0 & 45,0 & 27,0 & 43,0 & 46,3 & 47,0 & 46,7 \\
\hline Azúcares totales (\%) & 56,0 & 50,0 & 53,0 & 43,0 & 26,0 & 35,0 & 45,0 & 42,7 & 43,8 \\
\hline Humedad (\%) & 41,0 & 46,0 & 43,0 & 52,0 & 71,0 & 55,0 & 51,7 & 51,0 & 51,3 \\
\hline Energía (\%) & 3,47 & 2,76 & 3,04 & 2,81 & 1,62 & 1,31 & 2,70 & 2,30 & 2,50 \\
\hline Energía (kcal) & 236,0 & 219,0 & 228,0 & 190,0 & 115,0 & 181,0 & 193,0 & 196,7 & 194,8 \\
\hline $\begin{array}{l}\text { Capacidad } \\
\text { antioxidante (umol } \\
\text { ET/100g) }\end{array}$ & 4.399 & 6.544 & 6.787 & 6.155 & 7.461 & 7.929 & 6.216 & 6.876 & 6.546 \\
\hline $\begin{array}{l}\text { Flavonoides }(\mathrm{mg} \\
\text { CAT/100g) }\end{array}$ & 239 & 266 & 218 & 183 & 302 & 121 & 253 & 190 & 222 \\
\hline
\end{tabular}

Respecto de la capacidad antioxidante y el contenido de flavonoides de pulpa y semilla (Cuadro $\mathrm{N}^{\circ} 1$ ) se observa que contienen niveles de antioxidantes promedio $8,6 \%$ mayores en la semilla que en la pulpa, observándose diferencias entre el origen de las muestras analizadas, según comunidad y sector de procedencia. En el contenido de flavonoides se observa que existe una variación que va de 176 a $3.596 \mathrm{mg} \mathrm{CAT/100} \mathrm{g}$, registrando los menores valores para semilla y los mayores para pulpa. El arrope (Cuadro $\mathrm{N}^{\circ} 2$ ) mostró una cantidad de antioxidante que osciló entre 4.399 y 7.929 (umol ET/100 g) y flavonoides que variaron entre 121 y $302 \mathrm{mg} \mathrm{CAT/100} \mathrm{g}$, según origen de la muestra. Las diferencias registradas se podrían deber al origen y condición del material analizado y a diferencias en las condiciones de proceso del arrope.

La capacidad antioxidante ORAC se ha convertido en un estándar para determinar la capacidad de antioxidantes en alimentos, jugos y aditivos alimenticios. El Departamento de Agricultura de Estados Unidos (USDA) emite una lista sobre el valor ORAC de varios vegetales. Aunque los expertos sostienen que no hay suficiente evidencia científica, se puede proporcionar alguna orientación para comparar la magnitud antioxidante de un alimento con relación a otro. USDA recomienda una ingesta de entre 3.000 y 5.000 unidades ORAC diarias para combatir, mediante la alimentación, los posibles efectos de los radicales libres, por lo que los niveles presentados por las muestras superarían esos niveles (Navarro-González, 2017). También, los contenidos de antioxidantes encontrados en componentes de los frutos de chañar (Cuadro № 1), superan o están en el rango de los contenidos que registran hortalizas, como acelga, lechuga y frutas, como almendra con piel y arándano fresco (INTA, 2018).

Si se observa en el Cuadro $N^{\circ} 2$ la relación entre capacidad antioxidante y contenido de flavonoides en las muestras de arrope $(n=6)$, se constata que no hay una relación entre estos constituyentes del arrope del chañar. Daud et al. (2010) encontraron correlación entre contenido fenoles y actividad antioxidante, mientras que Palomino et al. (2009) encontraron una débil relación entre actividad antioxidante y contenido de flavonoides. Según los resultados de diversos autores (Zapata et al., 2013; Mazzeo et al, 2011; Anwar et al., 2008; Agostini et al., 2004) factores como la variabilidad de las muestras, los procesos y la interacción de compuestos pueden explicar esa relación. 
La efectividad antioxidante es mayoritariamente debido a la presencia de compuestos fenólicos. Silva et al. (1999) indicaron que los componentes de los carbohidratos son emolientes, mientras que los de los flavonoides son clasificados como entiespamódicos y antihistamínicos. Middleton et al. (2000) señalan que los flavonoides tienen actividad antiinflamatoria, antiviral, antibacterial y coadyuvante en la disminución del riesgo a padecer enfermedades cardiovasculares.

Reynoso et al. (2013) informan que el arrope es tradicionalmente preparado para tratamientos de enfermedades inflamatorias del sistema respiratorio, tales como bronquitis, laringitis y faringitis, caracterizadas por dolor y dificultad de deglución, tos constante y dolor en el pecho. El efecto analgésico del chañar se debería a las propiedades opioides de la planta, factor que sería responsable del efecto antitusivo atribuido a esta especie en la medicina popular, justificando también el uso de estos productos por sus significativos efectos antinociceptivos, que alteran aspectos sensoriales para disminuir la sensación de dolor. Tanto el arrope como los extractos acuosos derivados de los frutos del chañar, según los mismos autores, tienen un potente efecto antinociceptivo y constituyen un ejemplo de producto etnofarmacología.

\section{CONCLUSIONES}

Las comunidades de Piedra Colgada y San Pedro en la región de Atacama, se identifican plenamente con los chañares y con el uso histórico que ellos y sus antepasados han realizado de sus frutos. Son capaces de reconocer la importancia ancestral del arrope y harina de chañar, tanto en su dieta como en el ámbito de la salud, entendiendo la necesidad de traspasar sus conocimientos a la familia y cercanos como una forma de mantener y rescatar esta tradición, especialmente destacada como parte de la cultura Colla.

Del estudio también se desprende el interés y el reconocimiento por parte de la comunidad sobre generar cambios en su técnica de elaboración y de gestión comercial, de manera de adaptarse a nuevas condiciones de mercado y responder a nuevas demandas de consumidores, incluso fuera del ámbito local, buscando con ello valorizar su tradición y su trabajo, ya que una de las limitantes actuales es precisamente no ver reflejado en el precio de venta las horas de dedicación que requiere esta labor. Se abre así una importante oportunidad para desarrollar un modelo de negocios que pueda colaborar en el logro de este anhelo de la comunidad.

Sobre la base de la exploración realizada y de los aportes de diversos autores se puede señalar que el fruto del chañar tendría un alto potencial nutracéutico dado por el contenido de polifenoles, específicamente flavonoides, y también por la actividad antioxidante, permitiendo que estas características particulares sean utilizadas para promover el consumo del fruto de chañar y productos derivados, como arrope u otros.

La alta presencia de carbohidratos, flavonoides y antioxidantes, además de lo que la literatura indica, posiciona los frutos y derivados del chañar como un importante producto con potencial, no solamente en el ámbito del consumo de alimentos dirigidos a mercados nichos sino también en el ámbito de la farmacopea, especialmente en el ámbito de la medicina popular.

La identificación de usos potenciales derivados de las características de composición del fruto, además de los usos actuales recogidos, podrán configurar las fortalezas de la materia prima, que además pudiera ser parte del análisis estratégico de la unidad de negocio basada en productos con base en chañar.

Se puede afirmar que el fruto del chañar es un producto muy versátil, con un amplio rango potencial de usos en la industria alimentaria y medicinal, lo que queda de manifiesto en su composición nutricional. Esta drupa carnosa, comestible, de sabor dulce ya se emplea para la elaboración del arrope, que se obtiene mediante la cocción y concentración hasta lograr una apariencia viscosa similar a un jarabe o syrup, el cual es utilizado en la medicina popular para dolencias de la garganta y en la gastronomía. Sin embargo, sus características permiten ampliar su espectro de uso y podría ser utilizado para distintas preparaciones como dulces, flanes, tortas, postres espumosos con la adición de crema batida y gelatina sin sabor previamente hidratada y 
disuelta. A su vez su uso en la elaboración de salsas para acompañar helados y para endulzar bebidas amplía sus posibilidades de uso y, consecuentemente, de unidades potenciales de negocio.

\section{RECONOCIMIENTOS}

El presente estudio fue realizado con el apoyo de la Fundación para la Innovación Agraria (FIA) y con la valiosa colaboración de las Comunidades de la Región de Atacama.

\section{REFERENCIAS}

Agostini, L., Morón, M.; Ramón, A. y Ayala, A., 2004. Determinación de la capacidad antioxidante de flavonoides en frutas y verduras frescas y tratadas térmicamente. Archivos Latinoamericanos de Nutrición, 54(1):89-92.

Anwar, F.; Iqbal, S. and Sultana, B., 2008. Effect of different cooking methods on the antioxidant activity of some vegetables from Pakistan. International Journal of Food Science and Technology, 43, 560-567.

Daud, A., Reynoso, M.; Habib, N. y Sanchez, R., 2010. Antioxidant activity and acute oral toxicity of Phrygilanthus acutifolius flowers. Latin American Journal of Pharmacy, 29 (2010), pp. 783-788

Dewanto, B.; Wu, X.; Adom, K. K. and Liu, R. H., 2002. Thermal processing enhances the nutritional value of Tomatoes by increasing total antioxidant activity. Journal of Agricultural and Food Chemistry 50: 3010-3014.

FAO, 1999. Actividades forestales de la FAO. Hacia una definición uniforme de los productos forestales no madereros. En: http://www.fao.org/docrep/x2450s/x2450s0d.htm\#fao\%20forestry

Guorong, D.; Mingiun, L.; Fengwang, M. and Dong, L., 2009. Antioxidant capacity and the relationship with polyphenol and vitamin C in Actinidia fruits. Food Chemistry 113 (2009) 557-562

INTA, 2018. Instituto de Nutrición y Tecnología de los Alimentos. http://www.portalantioxidantes.com/base-dedatos-de-antioxidantes/\#Base\%20de\%20Datos\%20de\%20Antioxidantes\%20en\%20Frutas (Consultado 20 junio de 2018).

Kim, D.; Jeong, S. and Lee, C., 2003. Anioxidant capacity of phenolic phytochemicals from various cultivars of Plums. Food Chemistry. 81, 321-326.

Martins, C. P. C.; Ferreira, M. V. S.; Esmerino, E. A.; Moraes, J.; Pimentel, T. C.; Rocha, R. S.; Freitas, M. Q.; Santos, J. S.; Ranadheera, C. S.; Rosa, L. S.; Teodoro, A. J.; Mathias, S. P.; Silva, M.; Raices, R. S. L.; Couto, S. R. M.; Granato, D. and Cruz, A.G ., 2018. Chemical, sensory and functional properties of whey-based popsicles manufactured with Watermelon juice concentrated at different temperatures. Food Chemistry 255:58-66.

Masson, L., 2012. Semillas de frutos nativos y cultivados en Chile: Su aceite como fuente de compuestos nacionales. Universidad Complutense de Madrid, Facultad de Farmacia, Departamento de Nutrición y Bromatologia. Memoria para optar al grado de Doctor. $277 \mathrm{p}$.

Mazzeo, T.; N'Dri, D.; Chiavaro, E.; Fogliano, V.; Pellegrini, N. and Visconti, A., 2011. Effect of two cooking procedures on phytochemical compounds, total antioxidant capacity and colour of selected frozen vegetables. Food Chemistry, 128, 627-633.

Middleton, E. J. R.; Kandaswami, C. and Theoharides, T., 2000. The effects of plant flavonoids on mammalian cells: Implications for inflammation, heart disease and cancer. Pharmacological Reviews 52(4): 673-751.

Navarro-González, I.; Periago, M. J. and García-Alonso, F. J., 2017. Estimation of the antioxidant capacity of foods consumed by the Spanish population. Rev Chil Nutr 44 (2):183-188

Nogués, E.; Castro, O. E.; Correa, R. J.; Puricelli, M.; Gómez, H.; Beale, I. y López, R.. 2013. Revalorización del chañar (Geoffroea decorticans) en ambientes áridos y semiáridos de Argentina, múltiples usos y posibilidades agroindustriales para las comunidades campesinas. Revista de Divulgación Técnica Agrícola y Agroindustrial $45: 1-8$.

Orrabalis, C.; Gorostegui, H.; Calandri, E. and Guzmán, C., 2013. Functional parameters and glucose content in flours obtained from ripe fruit of Chañar (Geoffroea decorticans) from semi-arid and arid zones of Formosa 
Orrabalis, C.; Gorostegui, H.; Calandri, E. y Guzmán, C., 2014. Caracterización química y evaluación sensorial de dulces novedosos elaborados con frutos de Geoffroea decorticans, Fabaceae. Phyton 83.117-125.

Palomino, L. R.; García, C.; Gil, J.; Rojano, B. and Durango, D., 2009. Determination of phenolic content and evaluation of antioxidant activity of Propolis from Antioquia (Colombia). Vitae 16(3):388-395.

Reynoso, M. A.; Vera, N.; Aristimuño, M. E.; Daud, A. and Sánchez, A., 2013. Antinociceptive activity of fruits extracts and arrope of Geoffroea decorticans (Chañar). Journal of Ethnopharcacology 145:355-362.

Silva, R.; Ruiz, R. E. y Ruiz, S. O., 1999. Estudio fitoquímico de frutos de Geoffroea decorticans (Gill. Ex Hook. Et Arn.) Burk. Leguminosae (Fabaceae). Acta Farm. Bonaerense 18(3):217-219.

Zapata, K.; Cortés, F. y Rojano, B., 2013. Polifenoles y actividad antioxidante del fruto de guayaba agria (Psidium araca). Información Tecnológica 24(5):103-112. 